\title{
Effectiveness of scapula-focused approaches in patients with rotator cuff related shoulder pain: a systematic review and meta-analysis
}

Julie Bury PT, BSc ( $\left.{ }^{a}\right)$

a. Doncaster \& Bassetlaw Hospitals NHS Foundation Trust, Physiotherapy Dept., Doncaster Royal Infirmary, Doncaster, UK.

E-mail: julie.bury@nhs.net

Morgan West PT, MSc $\left({ }^{b}\right)$

b. Sherwood Forest NHS Foundation Trust, Kings Mill Hospital, Mansfield, UK.

E-mail: Morgan.West@sfh-tr.nhs.uk

Gema Chamorro-Moriana PT, PhD ( $\left.{ }^{\circ}\right)$

c. Physiotherapy Department. Faculty of Nursing, Physiotherapy and Podiatric. University of Seville. Departamento de Fisioterapia. Edificio de Fisioterapia y Podología. Universidad de Sevilla. Spain.

E-mail: gchamorro@us.es

Chris Littlewood PT, PhD $\left({ }^{\mathrm{d}, \mathrm{e}}\right)$ * Corresponding author

d. School of Health \& Related Research, University of Sheffield, Regent Court, 30 Regent Street, Sheffield, S1 4DA, UK.

e. Arthritis Research UK Primary Care Centre, Primary Care Sciences, Keele University, Staffordshire, ST5 5BG, UK

E-mail: c.littlewood@sheffield.ac.uk

Tel: +44114222 0888/ Fax: +441142724095 


\begin{abstract}
Background: Rotator cuff related shoulder pain (RCSP) is common with a range of conservative treatments currently offered. Evidence supporting superiority of one approach over another is lacking. Scapula focused approaches (SFA) are frequently prescribed and warrant investigation.

Objective: To evaluate the effectiveness of SFA in RCSP.

Design: Systematic review of randomised controlled trials.

Methods: An electronic search including MEDLINE, PEDro, ENFISPO to January 2016 was supplemented by hand searching. Randomised controlled trials were included; appraised using the PEDro scale and synthesised via meta-analysis or narratively, where appropriate. Results: Four studies $(n=190)$ reported on pain and three studies $(n=122)$ reported on disability. Regarding pain, there was statistical but not clinically significant benefit of SFA versus generalised approaches (mean difference (VAS) $0.714 ; 95 \% \mathrm{Cl} 0.402$ to 1.026 ) in the short term (<6 weeks); regarding disability, there was significant benefit of SFA versus generalised approaches (mean difference $14.0 ; 95 \% \mathrm{Cl} 11.2$ to 16.8) in the short term (<6 weeks). One study $(n=22)$ reported disability at 3 months, which was not statistically significant. Evidence is conflicting from four studies relating to the effect of SFA on scapula position/ movement.

Conclusion: SFA for RCSP confers benefit over generalised approaches up to six weeks but this benefit is not apparent by 3 months. Early changes in pain are not clinically significant. With regards to scapula position/ movement, the evidence is conflicting. These preliminary conclusions should be treated with significant caution due to limitations of the evidence base.
\end{abstract}

Keywords: scapula, rotator cuff, rehabilitation, exercise, systematic review. 


\section{Introduction}

Shoulder pain is a common musculoskeletal complaint, with a prevalence of $7-26 \%$ in the general population (1). Disorders of the rotator cuff are most frequently recorded as a source of these symptoms, reported in up to $70 \%$ of cases $(2,3)$. A range of terms including rotator cuff tendinopathy, shoulder impingement syndrome and subacromial pain are used to describe shoulder pain thought to be attributable to the rotator cuff but currently there is lack of consensus about the most appropriate terminology (4).

The resulting pain and loss of function secondary to rotator cuff disorders can be debilitating and impact on an individual's ability to self-care and work; posing a significant socioeconomic burden $(2,5)$. Conservative treatment, including exercise therapy, is recommended as the primary treatment; but there is considerable uncertainty relating to the relative effectiveness of such approaches (6-12), and as a result large variations in practice exist $(3,6,9,13,14)$. Furthermore, there is uncertainty relating to the role that altered scapula position and movement plays, termed scapula dyskinesis; which is widely regarded as a common finding with this condition (15-18). As a consequence, despite popularity, confusion exists over the value of scapula-focused approaches (SFA) within rehabilitation programmes (19).

In this context, the aim of this systematic review is to synthesise the evidence relating to the effectiveness of SFA for rotator cuff related shoulder pain (RCSP) with the objective of informing clinical practice.

\section{Methods}

These methods were pre-specified and recorded in a protocol, consistent with the PRISMA statement (20).

\section{Data sources and search strategy}

An electronic search of MEDLINE, CINAHL, PEDro, ENFISPO, LILIACS, IBECS and DIALNET was carried out from inception to January 2016. In addition to this, reference list checking of included studies was employed and consultation with experts was also utilised to verify any additional published or unpublished sources. Two examples of the search terms and keywords used for MEDLINE (Mesh terms, Medical Subject Headings) and for ENFISPO (DeCS terms, " Descriptores en Ciencias de la Salud"); are shown in table 1 and table 2 , respectively. 
Table 1. MEDLINE search strategy

\begin{tabular}{|c|c|c|c|}
\hline \multicolumn{4}{|c|}{ Search terms } \\
\hline 1 & $\begin{array}{l}\text { shoulder pain/ OR shoulder joint/ OR } \\
\text { shoulder impingement syndrome/ OR } \\
\text { subacromial pain syndrome*.mp. OR rotator } \\
\text { cuff/ }\end{array}$ & 11 & (scapula* adj2 rehabili*).mp. \\
\hline 2 & subacromial impingement syndrome*.mp. & 12 & 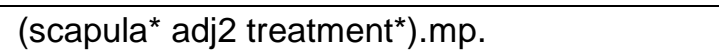 \\
\hline 3 & supraspinatus tend ${ }^{*} . \mathrm{mp}$ & 13 & scapula* focused.mp. \\
\hline 4 & shoulder burs*.mp. & 14 & scapula* approach*.mp. \\
\hline 5 & shoulder tend ${ }^{*} . \mathrm{mp}$. & 15 & (scapula* adj2 strength*).mp. \\
\hline 6 & painful arc*.mp. & 16 & motor control exercise*.mp. \\
\hline 7 & 1 OR 2 OR 3 OR 4 OR 5 OR 6 & 17 & $\begin{array}{l}8 \text { OR } 9 \text { OR } 10 \text { OR } 11 \text { OR } 12 \text { OR } 13 \text { OR } 14 \\
\text { OR } 15 \text { OR } 16\end{array}$ \\
\hline 8 & scapula/ OR scapula*.mp. & 18 & 7 and 17 \\
\hline 9 & scapula* stabil*.mp. & 19 & $\begin{array}{l}\text { limit } 18 \text { to ("all adult ( } 19 \text { plus years)" and } \\
\text { humans and (clinical trial or controlled clinical } \\
\text { trial or randomized controlled trial)) }\end{array}$ \\
\hline 10 & (scapula* adj2 exercise*).mp. & 20 & limit 19 to English language \\
\hline
\end{tabular}

Table 2. ENFISPO search strategy

\begin{tabular}{|c|l|c|l|}
\hline \multicolumn{2}{|l|}{ Search terms } \\
\cline { 1 - 2 } 1 & $\begin{array}{l}\text { dolor(F)hombro OR articulación(F)hombro } \\
\text { OR } \\
\text { síndrome(F)abducción(F)dolorosa(F)hombro } \\
\text { OR síndrome(F)hombro(F)doloroso OR } \\
\text { síndrome(F)impingement(F)hombro OR } \\
\text { síndrome(F)pinzamiento(F)hombro OR } \\
\text { dolor(F)subacromial OR manguito(F)rotador }\end{array}$ & 11 & escápula(F)terapéutica \\
\hline 2 & síndrome(F)pinzamiento(F)subacromial & 12 & escápula(F)tratamiento \\
\hline 3 & $\begin{array}{l}\text { tend }(F) \text { supraespinoso OR } \\
\text { síndrome(F)supraespinoso }\end{array}$ & 13 & escápula(F)rehabilitación \\
\hline 4 & bursitis(F)hombro & 14 & escápula(F)fisioterapia \\
\hline 5 & tend\$(F)hombro & 15 & escápula(F)musculación \\
\hline 6 & síndrome(F)arco(F)doloroso & 16 & $\begin{array}{l}8 \text { OR 9 OR 10 OR 11 OR 12 OR 13 OR 14 } \\
\text { OR 15 }\end{array}$ \\
\hline 7 & 1 OR 2 OR 3 OR 4 OR 5 OR 6 & 17 & 7 AND 16 \\
\hline 8 & escápula & 18 & $\begin{array}{l}\text { ensayo(F)clínico(F)controlado(F)aleatorio OR } \\
\text { ensayo(F)clínico(F)controlado(F)randomizado } \\
\text { OR ensayo(F)controlado(F)aleatorio }\end{array}$ \\
\hline 9 & estabilización(F)escápula & 19 & 7 AND 18 \\
\hline 10 & escápula(F)ejercicio & 20 & 16 AND 18 \\
\hline
\end{tabular}




\section{Study selection and inclusion criteria}

For a study to be included in this review, the following criteria had to be met:

Population - Adults with signs \& symptoms of RCSP (inclusive of terms such as rotator cuff tendinopathy, shoulder impingement syndrome), commonly described as unilateral shoulder pain, localised around the acromion; worse with overhead activity; usually full range of shoulder movement; a combination of positive impingement tests; presence of a painful arc; reproduction or worsening of pain on resisted muscle testing; and no cervical spine involvement $(4,12,21)$.

Interventions - SFA (including exercise therapy, stretches and/or manual therapy) with the aim to address the pain and disability found with RCSP and/or with the intention of changing scapula biomechanics, including position, movement, strength, motor control, and/or muscle length.

Comparison - Any comparison that adopts a general or non-scapula approach, such as usual care or an alternative exercise therapy/intervention that is not focused specifically on scapula biomechanics.

Outcomes - Any validated measures of patient-reported pain and/or disability; plus biomechanical outcomes relating to the scapula.

Study design - Randomised controlled trials (RCTs).

Language - Restricted to studies reported in English and Spanish.

Setting - Not limited to a particular setting.

The titles and abstracts of the search results were screened to determine if a study met the pre-specified inclusion criteria. Of these identified studies, and any with questions over their relevance, the full text article was sourced; the reasons for any exclusions at this stage were documented.

\section{Data extraction}

Data extraction was performed by one reviewer (JB) and checked for accuracy by a second reviewer (GCM) using a pre-designed table to detail information on study characteristics, participant characteristics, interventions, outcome measures and results.

\section{Quality appraisal}

Eligible studies were assessed on methodological quality using the Physiotherapy Evidence Database (PEDro) critical appraisal tool (22), which has been found to be valid and reliable for evaluating both the internal validity of a study (criteria 2-9), as well as the adequacy of the statistical information to interpret the results (criteria 10-11) (23-25). It consists of 11 criteria overall; although criterion 1 refers to the external validity of the trial and is not included in the final score (24). Each criterion is rated as Yes (1 point) or No (0 points), with a maximum score out of 10 ; a PEDro score $\geq 6 / 10$ is classed as a high quality study (26). Out of the 4 studies, 3 had already been scored on the PEDro database and this data was extracted. The remaining study (27) was appraised by one reviewer (JB) and verified by a second reviewer $(\mathrm{CL})$. 


\section{Data Synthesis}

Meta-analysis was conducted using OpenMetaAnalyst software (28). Due to the inherent heterogeneity within the literature, the DerSimonian and Laird random effects model was used (29). Statistical between study heterogeneity was assessed with the $\mathrm{I}^{2}$ statistic, and this review considered $25 \%$ low, $50 \%$ moderate and $75 \%$ high (30). Pain scores were measured on the same tool, the visual analogue scale (VAS), so mean pain scores along with their respective standard deviations (SD) were extracted and pooled. Disability scores were measured on different tools but these tools used the same scales (0 to 100) and it was felt that they measured similar constructs of shoulder related disability so, similar to pain outcomes, mean scores along with their respective standard deviations (SD) were extracted and pooled (31). The disability scale used in one study (32) required reversal of the direction of the scores to enable meta-analysis, accordingly the total scores of the scale were multiplied by -1 (33).

As a result of study heterogeneity relating to measurement of outcomes of scapula biomechanics, a narrative synthesis using a rating system for levels of evidence was used (34). This rating system, displayed in table 3, is used to summarise the results in which the quality and outcomes of individual studies are taken into account.

Table 3. Levels of evidence

\begin{tabular}{|l|l|}
\hline Strong evidence & Consistent findings among multiple high quality RCTs \\
\hline Moderate evidence & $\begin{array}{l}\text { Consistent findings among multiple low-quality RCTs and/or one high-quality } \\
\text { RCT }\end{array}$ \\
\hline Limited evidence & One low quality RCT \\
\hline Conflicting evidence & Inconsistent findings among multiple RCTs \\
\hline No evidence from trials & No RCTs \\
\hline
\end{tabular}

\section{Results:}

\section{Search results}

Searching of the electronic databases produced 464 records in total. A further 13 were identified through other sources: 1 through a Google search, 2 by contact with experts and 10 through reference list checking of the identified articles. Following the removal of duplicates, 437 articles were screened by title and abstract, and after exclusions 7 required the full-text to be assessed. Out of these, 3 were excluded for either not meeting the study design, participant or intervention criteria, resulting in 4 studies for inclusion in this review. Figure 1 shows the search and study selection process. 


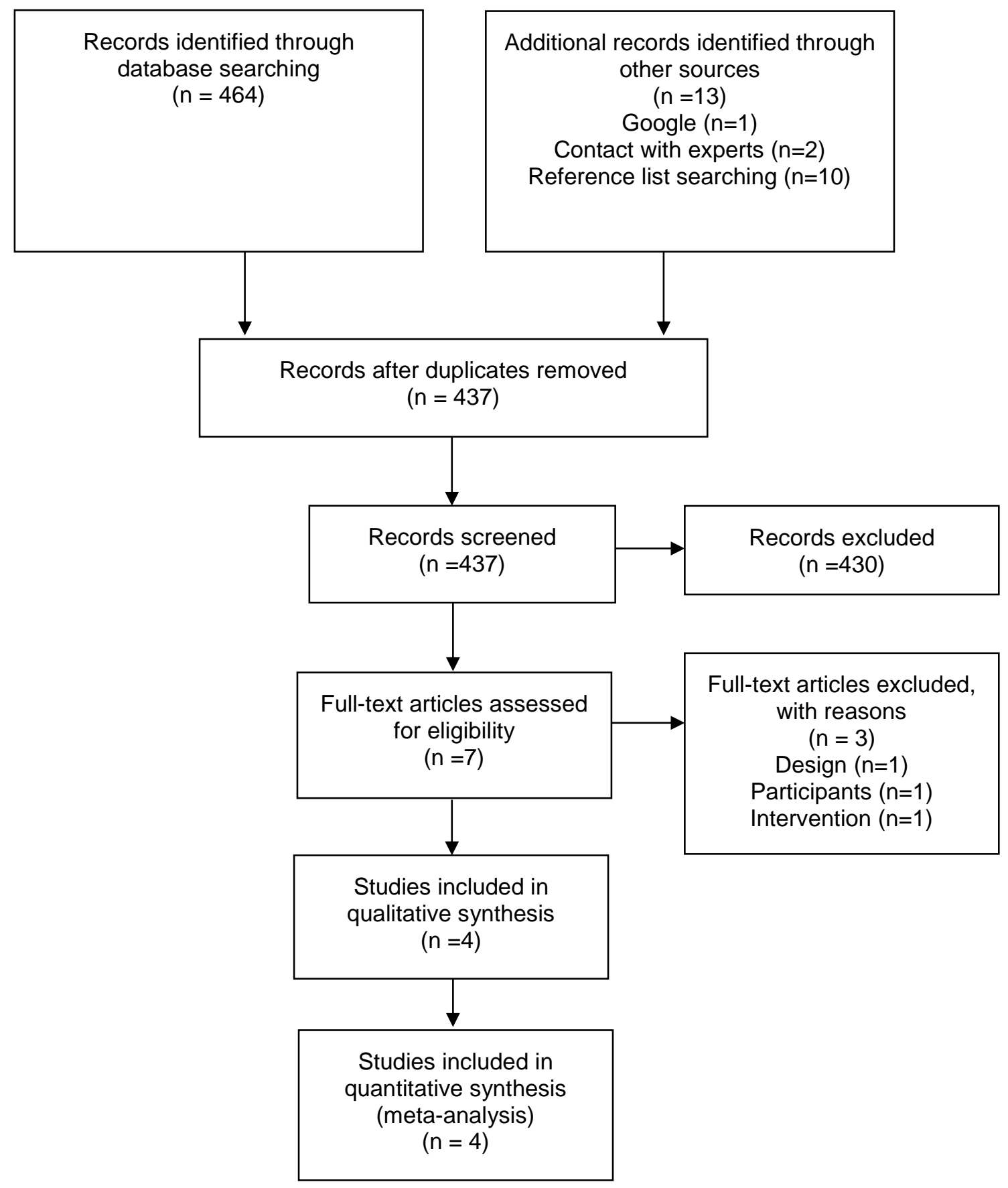

Figure 1. PRISMA flow diagram showing study selection process

\section{Quality assessment}

The results of the PEDro scoring are shown in table 4. Overall, two out of the four studies were considered to be of high quality. Random allocation was apparent in all the studies however allocation concealment was not featured in any of them. There was a consistent lack of blinding of subjects or therapists, but two studies had blinded assessors of some outcomes. Intention-to-treat (ITT) analysis was only evident in one study. 
Table 4. Completed PEDro quality appraisal

\begin{tabular}{|c|c|c|c|c|c|c|c|c|c|c|c|c|}
\hline \multirow[b]{2}{*}{ Study: } & \multicolumn{11}{|c|}{ Criteria: } & \multirow[b]{2}{*}{ Total score } \\
\hline & 1 & 2 & 3 & 4 & 5 & 6 & 7 & 8 & 9 & 10 & 11 & \\
\hline Baskurt et al (32) & $x$ & $\checkmark$ & $x$ & $\checkmark$ & $x$ & $x$ & $x$ & $\checkmark$ & $x$ & $\checkmark$ & $\checkmark$ & 5 \\
\hline Moezy et al (36) & $\checkmark$ & $\checkmark$ & $x$ & $\checkmark$ & $x$ & $x$ & $\checkmark$ & $\checkmark$ & $x$ & $\checkmark$ & $\checkmark$ & 6 \\
\hline Struyf et al (35) & $\checkmark$ & $\checkmark$ & $x$ & $\checkmark$ & $x$ & $x$ & $\checkmark$ & $\checkmark$ & $\bar{\checkmark}$ & $\checkmark$ & $\checkmark$ & 7 \\
\hline Shah et al (27) & $\checkmark$ & $\checkmark$ & $x$ & $\checkmark$ & $x$ & $x$ & $x$ & $\checkmark$ & $x$ & $\checkmark$ & $\checkmark$ & 5 \\
\hline
\end{tabular}

1, Eligibility criteria were specified. 2, Subjects were randomly allocated to groups. 3, Allocation was concealed. 4, Groups were similar at baseline regarding the most important prognostic indicators. 5, There was blinding of all subjects. 6, There was blinding of all therapists who administered the therapy. 7, There was blinding of all assessors who measured at least one key outcome. 8, Measures of at least one key outcome were obtained from more than $85 \%$ of the subjects initially allocated to groups. 9, All subjects for whom outcome measures were available received the treatment or control condition as allocated or, where this was not the case data for at least one key outcome was analyzed by 'intention-to-treat'. 10, The results of between-group statistical comparisons are reported for at least one key outcome. 11, The study provides both point measures and measures of variability for at least one key outcome). $\checkmark=$ criteria met; $x=$ criteria not met

\section{Study \& participant characteristics}

A detailed summary of the characteristics and results of each study are shown in table 5 .

A total of 190 adults (mean age 47.9 years) were included through convenience sampling across the four studies, comprising 67 males and 123 females. The studies were conducted in India (27), Belgium (35), Turkey (32) and Iran (36).

\section{Interventions/comparisons}

All four studies evaluated a SFA compared to a more generalised approach, however this varied in its content. Two studies were very similar and compared what they described as conventional physiotherapy to the same programme, but with the addition of scapula stabilisation exercises in the intervention group $(27,32)$. The latter study also had an advice component (32). One study had an intervention group of mobilisations, stretching and motor control exercises all focused on the scapula, compared to glenohumeral joint passive mobilisations with eccentric exercises for the rotator cuff (35); one study compared a scapula stabilisation based exercise therapy group of mainly exercises, stretches and advice to a physical therapy group which included range of movement exercises (ROM) and electrotherapy modalities (36). 
Table 5. Characteristics of included studies

\begin{tabular}{|c|c|c|c|}
\hline $\begin{array}{c}\text { Study } \\
\text { Characteristics }\end{array}$ & $\begin{array}{c}\text { Participant } \\
\text { Characteristics }\end{array}$ & Interventions \& Comparators & $\begin{array}{c}\text { Outcome Measures } \\
\text { and Results }\end{array}$ \\
\hline $\begin{array}{l}\text { Shah et al (27 ) } \\
\text { RCT - Comparing } \\
\text { the effectiveness } \\
\text { of conventional } \\
\text { physiotherapy } \\
\text { plus scapula } \\
\text { stability exercises } \\
\text { to conventional } \\
\text { physiotherapy } \\
\text { alone } \\
\text { Conducted in } \\
\text { India }\end{array}$ & $\begin{array}{l}\mathrm{n}=60 \\
\text { Convenience sampling } \\
\text { Sex }=31 \text { males }(52 \%) \text {; } \\
29 \text { females }(48 \%) \\
\text { Age(mean) = 46.93 } \\
\text { years } \\
\text { Main inclusion criteria: } \\
\text { - Age } 20-60 \text { years } \\
\text { - Pain with resisted } \\
\text { isometric abduction } \\
\text { - Painful arc of } \\
\text { movement between } \\
60 \text { - } 120 \text { degrees } \\
\text { - Painful palpation of } \\
\text { the rotator cuff } \\
\text { tendons } \\
\text { - Positive Neer sign \& } \\
\text { Hawkin's test }\end{array}$ & $\begin{array}{l}\text { Intervention Group }(\mathrm{n}=30) \text { : } \\
\text { conventional treatment } \\
\text { (strengthening exercises for } \\
\text { shoulder flexors, abductors, } \\
\text { horizontal abductors, external } \\
\text { rotators; stretches of pectoralis } \\
\text { major, levator scapulae, cross- } \\
\text { chest stretch, wand exercises \& } \\
\text { pendulum exercises); plus } \\
\text { scapula stability exercises } \\
\text { (scapula clock exercises, towel } \\
\text { sliding exercise, lawnmower } \\
\text { exercise, prone horizontal } \\
\text { abduction, press-up plus, wall } \\
\text { push-up \& scapula PNF with } \\
\text { weight shifting) - } 3 \text { sets of } 8 \\
\text { repetitions } \\
\text { Control group ( } \mathrm{n}=30 \text { ): } \\
\text { conventional Rx only (as above) } \\
\text { Frequency \& duration: daily, } 6 \\
\text { days/week for } 4 \text { weeks } \\
\text { Supervised exercises in a hospital } \\
\text { setting }\end{array}$ & $\begin{array}{l}\text { Outcomes assessed at } 4 \\
\text { weeks: } \\
\text { Visual analogue scale } \\
\text { (VAS): } 4.01 \text { (SD 0.65) vs. } \\
4.78 \text { (SD 0.76) reported } \\
\text { as statistically significant } \\
\text { in favour of intervention } \\
\text { group (p<0.0001). } \\
\text { Shoulder pain \& disability } \\
\text { index (SPADI): } 31.8 \text { (SD } \\
6.19 \text { ) vs. } 46.06 \text { (SD } 6.08) \\
\text { reported as statistically } \\
\text { significant in favour of } \\
\text { intervention group } \\
\text { (p<0.0001) } \\
\text { Lateral Scapula Slide test } \\
\text { (LSST): } 96.1 \text { mm (SD } \\
18.85 \text { ) vs. 101.13mm (SD } \\
13.94 \text { ) reported as } \\
\text { statistically significant in } \\
\text { favour of intervention } \\
\text { group (p<0.0001) }\end{array}$ \\
\hline $\begin{array}{l}\text { Struyf et al (35) } \\
\text { RCT - Comparing } \\
\text { the effectiveness } \\
\text { of a scapula - } \\
\text { focused treatment } \\
\text { with a control } \\
\text { Blinded assessor } \\
\text { Conducted in } \\
\text { Belgium }\end{array}$ & $\begin{array}{l}\mathrm{n}=22 \\
\text { Convenience sampling } \\
\text { Sex = } 10 \text { males }(45 \%) ; \\
12 \text { females }(55 \%) \\
\text { Age (mean) = 45.8 } \\
\text { years } \\
\text { Main inclusion criteria: } \\
\text { - Age >18 years } \\
\text { - Symptoms > } 30 \text { days } \\
\text { - Hawkin's test; Neer } \\
\text { test \& Jobe test: } 2 / 3 \\
\text { needed to be positive }\end{array}$ & $\begin{array}{l}\text { Intervention group- SFA protocol } \\
\text { ( } n=10 \text { ): passive manual } \\
\text { mobilisation to scapula; stretching } \\
\text { for levator scapulae, rhomboids \& } \\
\text { pectoralis minor; emphasis on a } \\
\text { scapula orientation exercise \& } \\
\text { scapula motor control training } 10 \\
\text { reps x } 1 / \text { day (lower trapezius } \\
\text { overhead arm lift in prone, middle } \\
\text { trapezius arm lift in } 90^{\circ} \text { abduction, } \\
\text { serratus anterior on all } 4 \text { 's with } \\
\text { weight transference or in side } \\
\text { lying with scapula protraction at } \\
90^{\circ} \text { elevation) } \\
\text { Control group ( } n=12) \text { : muscle } \\
\text { frictions ( } 5 \text { mins); passive } \\
\text { glenohumeral mobilisations ( } 5 \\
\text { mins); eccentric rotator cuff } \\
\text { training with resistance band } \\
3 \times 15 \text { reps; } 1 \text { x/day; ultrasound } \\
\text { therapy ( } 5 \text { minutes) } \\
\text { Progressed as able with load, } \\
\text { range, gravity, speed, no. of reps. } \\
\text { Frequency \& duration: } 30 \text { min } \\
\text { sessions between } 1-3 \times \text { / week }\end{array}$ & $\begin{array}{l}\text { Outcomes assessed post } \\
\text { treatment \& } 3 \text { months } \\
\text { Shoulder disability } \\
\text { questionnaire (SDQ): } 35.0 \\
\text { (SD 14.0) vs. } 48.7 \text { (SD } \\
\text { 11.3) reported as } \\
\text { statistically significant in } \\
\text { favour of intervention } \\
\text { group ( } p=0.025) \text { at the } \\
\text { end of treatment. } \\
\text { SDQ 15.6 vs. } 21.7 \text { at } 3 \\
\text { months, reported as not } \\
\text { significant } \\
\text { VAS: } 1.3 \text { SD (1.5) vs. } 2.3 \\
\text { (SD } 2.6) ; \text { statistical } \\
\text { significance not reported } \\
\text { Scapula outcomes: visual } \\
\text { observation; shoulder } \\
\text { posture; pectoralis minor } \\
\text { length (PML); upward } \\
\text { rotation range; kinetic } \\
\text { medial rotation test } \\
\text { reported as no statistically }\end{array}$ \\
\hline
\end{tabular}




\begin{tabular}{|c|c|c|c|}
\hline & & $\begin{array}{l}\text { (total of } 9 \text { sessions) } \\
\text { Supervised in a private setting \& } \\
\text { home programme }\end{array}$ & $\begin{array}{l}\text { significant difference } \\
\text { between groups }(p>0.05)\end{array}$ \\
\hline $\begin{array}{l}\text { Baskurt et al (32) } \\
\text { RCT - comparing } \\
\text { effectiveness of } \\
\text { scapula } \\
\text { stabilisation } \\
\text { exercise with a } \\
\text { control } \\
\text { Conducted in } \\
\text { Turkey }\end{array}$ & $\begin{array}{l}\mathrm{n}=40 \\
\text { Unclear sampling } \\
\text { method } \\
\text { Sex = } 13 \text { males (32\%); } \\
27 \text { females (68\%) } \\
\text { Age (mean) = 51 years } \\
\text { Main inclusion criteria: } \\
\text { - Positive Neer, } \\
\text { Hawkin's \& Jobe } \\
\text { tests } \\
\text { - Confirmed by } \\
\text { radiography \& } \\
\text { ultrasonography } \\
\text { Clinical diagnosis } \\
\text { made by an } \\
\text { orthopaedic surgeon }\end{array}$ & $\begin{array}{l}\text { Intervention group ( } \mathrm{n}=20 \text { ): Same } \\
\text { as group I, plus scapula } \\
\text { stabilisation exercises (scapula } \\
\text { PNF, scapula clock exercise, } \\
\text { weight-bearing exercises, scapula } \\
\text { depression, wall push-up, wall } \\
\text { slide exercise) } \\
\text { Progressed with reps to } 3 \times 10 \text {, } \\
\text { then increased resistance } \\
\text { Control group ( } \mathrm{n}=20 \text { ): flexibility } \\
\text { exercises (capsular stretches, } \\
\text { flexion and abduction ROM, } \\
\text { internal rotation stretches); } \\
\text { strengthening exercises (rotator } \\
\text { cuff \& deltoid); Codman exercises; } \\
\text { education \& advice } \\
\text { Frequency \& duration: } 3 \text { x week, } \\
\text { for } 6 \text { weeks }\end{array}$ & $\begin{array}{l}\text { Outcomes assessed at } 6 \\
\text { weeks } \\
\text { VAS: } 0.85 \text { (SD 1.08) vs. } \\
1.40 \text { (SD 1.78) reported } \\
\text { as not statistically } \\
\text { significant ( } p>0.05) \\
\text { Western Ontario Rotator } \\
\text { Cuff Index (WORC): } \\
82.61 \text { (SD 10.33) vs. } \\
70.82 \text { (SD 19.7) reported } \\
\text { as not statistically } \\
\text { significant ( } p>0.05 \text { ) } \\
\text { LSST: } 10 m m \text { (SD 6.2) vs. } \\
\text { 3mm (SD 2.9) reported as } \\
\text { statistically significant in } \\
\text { favour of intervention } \\
\text { group ( } p<0.0001 \text { ) }\end{array}$ \\
\hline $\begin{array}{l}\text { Moezy et al (36) } \\
\text { RCT - comparing } \\
\text { the effectiveness } \\
\text { of scapula } \\
\text { stabilisation based } \\
\text { exercise therapy } \\
\text { and physical } \\
\text { therapy } \\
\text { Conducted in Iran }\end{array}$ & $\begin{array}{l}\mathrm{n}=68 \\
\text { Convenience sampling } \\
\text { Sex }=13 \text { males (19\%); } \\
55 \text { females (81\%) } \\
\text { Age (mean) = 47.96 } \\
\text { years } \\
\text { Main inclusion criteria: } \\
\text { - } 18-75 \text { years } \\
\text { - Unilateral shoulder } \\
\text { pain > } 1 \text { month, } \\
\text { localised to the } \\
\text { acromion } \\
\text { - Painful palpation of } \\
\text { the rotator cuff } \\
\text { tendons } \\
\text { - Positive impingement } \\
\text { test (Hawkin's, Neer } \\
\text { or Empty can test) or } \\
\text { a painful arc ( } 60 \text { - } \\
\left.120^{\circ}\right) \\
\text { - Pain reproduced } \\
\text { during flexion }+/ \text { or } \\
\text { abduction }\end{array}$ & $\begin{array}{l}\text { Intervention group - scapula } \\
\text { stabilisation based exercise } \\
\text { therapy ( } n=33 \text { ): } 10 \text { min warm-up } \\
\text { on treadmill; stretches (sleeper } \\
\text { stretch, crossed arm stretch, } \\
\text { corner stretch, pectoral stretch); } \\
\text { strengthening exercises } \\
\text { (Theraband for rotator cuff, } \\
\text { scapula retractors, external } \\
\text { rotators and serratus anterior } \\
\text { punches); scapula stabilisation } \\
\text { exercises (Swiss ball T to Y to W, } \\
\text { scapula clock exercise); postural } \\
\text { ex's; advice } \\
\text { Progressed with resistance band } \\
\text { as able. Reps } 3 \times 10 \\
\text { Control group - physical therapy } \\
\text { group ( } n=35 \text { ): pendulum \& ROM } \\
\text { exercises; infra-red therapy, } \\
\text { ultrasound therapy \& TENS. } \\
\text { Frequency \& duration: } 3 \times \text { week, } \\
\text { for } 6 \text { weeks } \\
\text { Supervised exercises in a hospital } \\
\text { setting }\end{array}$ & $\begin{array}{l}\text { Outcomes assessed at } 6 \\
\text { weeks } \\
\text { VAS: } 2.76 \text { (SD 2.16) vs. } \\
3.14 \text { (SD 2.08); statistical } \\
\text { significance not reported } \\
\text { No measure of disability } \\
\text { or quality of life } \\
\text { Change score for scapula } \\
\text { position related outcomes: } \\
\text { statistically significant } \\
\text { between group } \\
\text { differences (p<0.001) in } \\
\text { scapula protraction, } \\
\text { forward shoulder } \\
\text { translation and pectoris } \\
\text { minor length; scapula } \\
\text { rotation and symmetry } \\
\text { were not statistically } \\
\text { significant ( } p=0.183 \text { \& } \\
0.578 \text { respectively) }\end{array}$ \\
\hline
\end{tabular}


The settings varied with two studies based in a hospital setting $(27,36)$; one in a private setting (35) and one unclear (32). All studies had a supervised programme $(27,32,36)$ and only one study also included home-based exercises (35), although there was no data provided on adherence to this.

The frequency and duration of these programmes also differed from three times per week for six weeks (a total of 18 sessions) $(32,36)$; to once a day, six days a week over four weeks (a total of 24 sessions) (27) and once a day, between 1-3 times a week (for a total of nine sessions), which also included the home exercise programme (35).

\section{Outcome measures/results}

To assess pain and disability all four studies used the Visual Analogue Scale (VAS) $(27,32,35,36)$. Measures of disability were the Shoulder Pain and Disability Index (SPADI) (27); the Shoulder Disability Questionnaire (SDQ) (35); and the Western Ontario Rotator Cuff (WORC) index (32). To assess scapula dyskinesis two studies used the Lateral Scapula Slide Test (LSST) (27,32); one used visual observation of scapula position (35); one study used scapula symmetry (36); and two used scapula ROM (35,36). Other biomechanical measures included assessment of muscle strength (32), motor control (kinetic medial rotation test) (35); pectoralis minor length (PML) and forward shoulder postures $(35,36)$. With regards to timings, three out of four studies assessed their outcomes pre and post-treatment, which ranged from $4-6$ weeks $(27,32,36)$ and only 1 study included a later follow-up period at 3 months post treatment (35).

\section{Data synthesis}

Four studies $(27,32,35,36)$ reported post treatment effect on pain from 190 participants; there was statistical but not clinically significant benefit of SFA versus generalised approaches (mean difference (VAS) $0.7 ; 95 \% \mathrm{Cl} 0.4$ to 1.0 ) in the short term (< 6 weeks) (figure 2).

Three studies $(27,32,35)$ reported post treatment effect on disability from 122 participants; there was statistical and clinically significant benefit of SFA versus generalised approaches (mean difference 14.0; 95\% Cl 11.2 to 16.8) in the short term ( $<6$ weeks) (figure 3).

Between study heterogeneity was negligible $\left(I^{2}=0 \%\right.$ and $0 \%$ respectively).

One study (35) reported post treatment effect on disability from 22 participants at three months; the difference between SFA versus a generalised approach was reported as not statistically significant. 

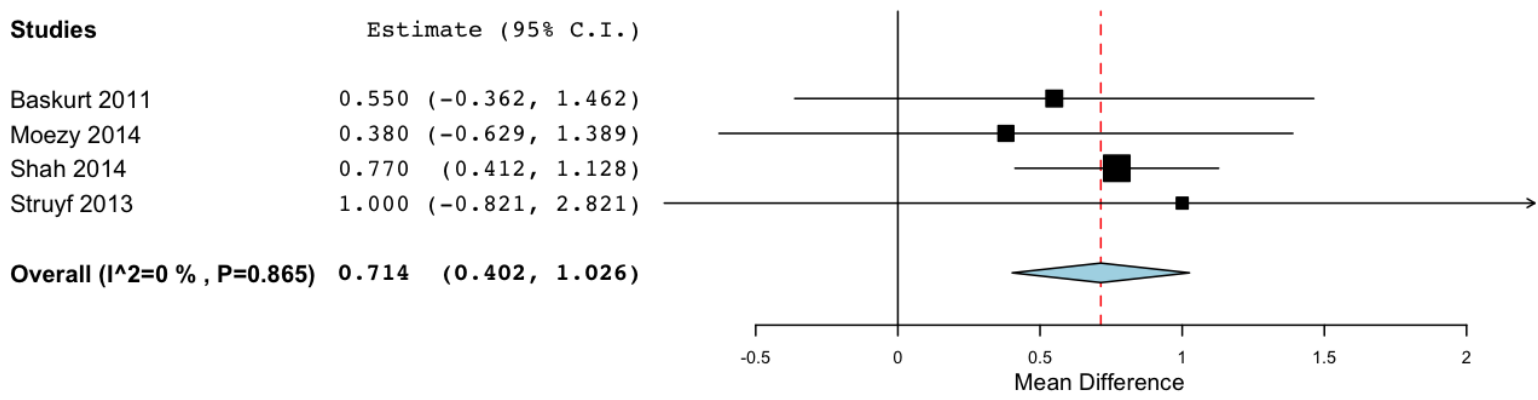

Figure 2 Forest plot of scapula focused approaches versus generalised approaches on short term pain $(<6$ weeks) *positive value favours scapula focused approaches

Studies

Baskurt 2011

Shah 2014

Struyf 2013

Overall $\left(\mathrm{I}^{\wedge} 2=0 \%, \mathrm{P}=0.893\right)$
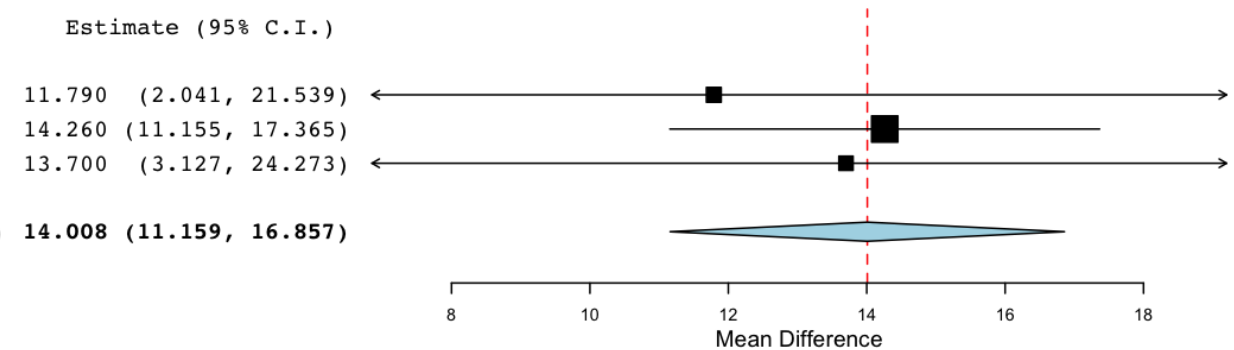

Figure 3 Forest plot of scapula focused approaches versus generalised approaches on short term function $(<6$ weeks) *positive value favours scapula focused approaches

Four studies $(27,32,35,36)$ reported post treatment effect on scapula position/ movement from 190 participants; there was conflicting evidence (inconsistent findings among multiple RCTs) of SFA versus generalised approaches. Studies at high risk of bias $(27,32)$ reported significant between group changes in favour of SFA whereas the studies at low risk of bias $(35,36)$ tended to report no significant between group difference. However, one study (36), regarded as presenting a low risk of bias, reported conflicting findings depending on the measure of scapula position/ movement reported.

\section{Discussion:}

This review summarises the effectiveness of SFA in patients with RCSP and suggests that SFA confers benefit over generalised approaches up to six weeks post commencement of treatment. Although these early changes in pain are statistically significant they are not regarded as clinically significant; early changes in disability are statistically and clinically significant but data from the only study that reported data beyond six weeks suggests the comparative benefit, in terms of disability, is no longer apparent by three months. Findings relating to the effect of SFA with regards to scapula position/ movement are unclear and current evidence is conflicting. 
Although the findings of this review suggest potential promise of SFA, we suggest caution with regards to interpretation. In relation to effect on pain, these findings are similar to those found in similar core stability or motor control approaches in the lumbar spine; small and clinically insignificant (31). In relation to effect on disability, these findings are based on three studies, two of which are regarded as presenting a high risk of bias $(27,32)$. Furthermore, the third study (35), although regarded as presenting a low risk of bias presents an interesting result that warrants further investigation. The mean change in the intervention group is 20.9 points on the SDQ, which is regarded as clinically significant, but only 2.2 points in the control group, despite prescription of active interventions in the control group. Such a small change in the control group is interesting and not in keeping with most, if not all, studies exploring the effects of active interventions in this field (37). The authors suggest possible reasons for this including potential therapist bias; because both treatments were delivered by the same physiotherapist (35). The influence of therapist beliefs has been recognised in other studies $(38,39)$. Hence, further high quality research designed to address these issues, for example including a wide range of physiotherapists, might significantly alter the preliminary inferences from this review.

Notwithstanding the above limitations, if the early beneficial response to SFA was regarded as true then this might suggest a potential ordering effect, i.e. a need to include different exercise prescriptions at different stages of the treatment pathway. Mulligan et al (40) explored this idea in a RCT where participants were randomly allocated to a 4-week program of scapula stabilisation exercises while the other group began with rotator cuff strengthening exercises. After four week the groups crossed over. These authors reported no significant difference between groups at any time point up to the final follow-up at eight weeks. Hence, the potential ordering effect is open to question.

This review suggested conflicting evidence with regards to effect of SFA on scapula position/ movement in comparison to generalized approaches. Clearly such conflict might result for a range of reasons; reliability of measurement of scapula position/ movement is notoriously poor (41) which could result in significant error when drawing such conclusions. Furthermore, some authors have begun to question the relevance of scapula position/ movement in RCSP (42) while others have suggested that the mechanism of action of SFA might not be related to a change in scapula position/ movement (43).

\section{Limitations of the included studies}

Despite the encouraging findings, there are some weaknesses within these studies that should be taken into account. In addition to the potentially spurious finding discussed above in relation to Struyf et al (35), limitations of the included studies include lack of allocation concealment, heterogeneity in term of outcome measurement, only reporting short term outcome, lack of intention-to-treat analysis and using a very small number, typically, one physiotherapist to deliver both intervention and comparator treatments which can result in preference bias. Although a lack of blinding is a feature of this body of evidence, such a design feature is typically elusive in pragmatic RCTs of complex therapeutic interventions, particularly when patient reported outcomes are the primary outcome measure. Hence, although a limitation of the studies, this should be considered in the wider context of this body of literature. 


\section{Strengths and limitations of this review}

This review has added insight that, to the author's knowledge, has not been previously reported. This review was undertaken in accordance with published guidelines by a team of reviewers with more than one member involved at each stage to minimise bias. This, in addition to the extensive and transparent search strategy, is a strength of this review. Unpublished studies were not identified for inclusion. Some authors have suggested that identifying unpublished studies for inclusion is important to minimise publication bias (44). To the contrary, others have questioned this suggesting that many unpublished studies eventually become published and truly unpublished studies might have poor or unclear methodology, which in turn might serve to introduce bias to the review (45). It has been suggested that it might be preferable to devote time to regularly updating reviews to capture studies when they are published (45). It is difficult to determine whether a lack of unpublished studies is a weakness of this review and whether inclusion, if available, would alter the conclusions drawn.

Finally, the findings from a systematic review are necessarily derived from the studies included. With clear methodological concerns, as described above, it is likely that the findings from this review will evolve as high quality studies become available.

\section{Implications for practice and further research}

SFA are widely used in the treatment of RCSP but there are significant limitations with the current evidence base and hence a clear clinical steer in relation to the optimal conservative treatment approach is not forthcoming at this time. We suggest that clinicians should balance decision-making with consideration of their own treatment preferences, the preferences of their patients, the complexity of SFA versus the utility of generalised approaches in tandem with the likely mix that will foster greatest therapeutic alliance and adherence to the chosen programme.

Hence, high quality, adequately powered, RCTs addressing the issues of allocation concealment are recommended, with suggestions to include longer-term follow-up, more homogeneity in the selection of outcome measures, and data analysis using the principles of ITT to facilitate firmer conclusions.

\section{Conclusions:}

This review summarises the effectiveness of SFA in patients with RCSP and suggests that SFA confers benefit over generalised approaches up to six weeks post commencement of treatment. Although these early changes in pain are statistically significant they are not regarded as clinically significant and data from the only study that reported data beyond six weeks suggests the benefit in terms of disability is not apparent by three months. Findings relating to the effect of SFA with regards to scapula position/ movement are unclear and current evidence is conflicting. These preliminary conclusions should be treated with caution due to significant methodological limitations and concerns regarding the validity of the evidence base. It is likely that future high quality primary studies will challenge these preliminary conclusions. 
Funding: JB was funded by a National Institute for Health Research Masters in Clinical Research Studentship

Conflict of interest: None 


\section{References:}

1. Luime J, Koes B, Hendriksen I, Burdorf A, Verhagen A, Miedema H, et al. Prevalence and incidence of shoulder pain in the general population; a systematic review. Scand J Rheumatol [Internet]. 2004;33(2):73-81.

2. Van der Windt DA, Koes BW, Boeke AJ, Devillé W, De Jong BA, Bouter LM. Shoulder disorders in general practice: prognostic indicators of outcome. $\mathrm{Br} \mathrm{J}$ Gen Pract [Internet]. 1996;46(410):519-23.

3. Michener $L$ a, Walsworth MK, Burnet EN. Effectiveness of rehabilitation for patients with subacromial impingement syndrome: a systematic review. J Hand Ther. 2004;17(2):152-64.

4. Carr A, Rees J. Subacromial Shoulder Pain . Pathw Guidel Subacromial Shoulder Pain. 2012;(September).

5. Harkness EF, Macfarlane GJ, Nahit ES, Silman AJ, McBeth J Mechanical and psychosocial factors predict new onset shoulder pain: a prospective cohort study of newly employed workers. Occup Environ Med. 2003;(60):850-7.

6. Hallgren HCB, Holmgren T, Oberg B, Johansson K, Adolfsson LE. A specific exercise strategy reduced the need for surgery in subacromial pain patients. Br J Sports Med [Internet]. 2014;48(19):1431-6.

7. Ylinen J, Vuorenmaa M, Paloneva J, Kiviranta I, Kautiainen H, Oikari M, et al. Exercise therapy is evidence-based treatment of shoulder impingement syndrome. Current practice or recommendation only. Eur J Phys Rehabil Med [Internet]. 2013;49(4):499-505.

8. Hanratty CE, McVeigh JG, Kerr DP, Basford JR, Finch MB, Pendleton A, et al. The Effectiveness of Physiotherapy Exercises in Subacromial Impingement Syndrome: A Systematic Review and Meta-Analysis. Semin Arthritis Rheum. 2012;42(June):297316.

9. Kuhn JE. Exercise in the treatment of rotator cuff impingement: A systematic review and a synthesized evidence-based rehabilitation protocol. J Shoulder Elb Surg. 2009;18(1):138-60.

10. Kromer TO, Tautenhahn UG, de Bie RA, Staal JB, Bastiaenen CHG. Effects of physiotherapy in patients with shoulder impingement syndrome: a systematic review of the literature. J Rehabil Med [Internet]. 2009;41(11):870-80.

11. Dong W, Goost H, Lin X-B, Burger C, Paul C, Wang Z-L, et al. Treatments for shoulder impingement syndrome: a PRISMA systematic review and network metaanalysis. Medicine (Baltimore) [Internet]. 2015;94(10):e510.

12. Littlewood C, Ashton J, Chance-Larsen K, May S, Sturrock B. Exercise for rotator cuff tendinopathy: A systematic review. Physiotherapy; 2012;98(2):101-9.

13. Dorrestijn O, Stevens M, Diercks RL, van der Meer K, Winters JC. A new interdisciplinary treatment strategy versus usual medical care for the treatment of subacromial impingement syndrome: a randomized controlled trial. BMC Musculoskelet Disord. 2007;8:15.

14. Littlewood C, Malliaras $P$, Chance-Larsen K. Therapeutic exercise for rotator cuff tendinopathy. Int J Rehabil Res [Internet]. 2015;(January 2016):1. 
15. Ludewig PM, Braman JP. Shoulder impingement: Biomechanical considerations in rehabilitation. Man Ther [Internet]. Elsevier Ltd; 2011;16(1):33-9.

16. Kibler W Ben, Ludewig PM, McClure PW, Michener L a, Bak K, Sciascia AD. Clinical implications of scapular dyskinesis in shoulder injury: the 2013 consensus statement from the "Scapular Summit". Br J Sports Med [Internet]. 2013;47(14):877-85.

17. Cools AM, Witvrouw EE, Declercq G a, Danneels L a, Cambier DC. Scapular muscle recruitment patterns: trapezius muscle latency with and without impingement symptoms. Am J Sports Med. 2003;31(4):542-9.

18. Lukasiewicz a C, McClure P, Michener L, Pratt N, Sennett B. Comparison of 3dimensional scapular position and orientation between subjects with and without shoulder impingement. J Orthop Sports Phys Ther. 1999;29(10):574-83; discussion 584-6.

19. Kibler WB. The role of the scapula in athletic shoulder function. Am J Sports Med. 1998;26(2):325-37.

20. Liberati A, Altman DG, Tetzlaff J, Mulrow C, Gøtzsche PC, loannidis JPA, et al. The PRISMA Statement for Reporting Systematic Reviews and Meta-Analyses of Studies That Evaluate Health Care Interventions: Explanation and Elaboration. PLoS Med [Internet]. 2009;6(7):e1000100.

21. Diercks R, Bron C, Dorrestijn O, Meskers C, Naber R, de Ruiter T, et al. Guideline for diagnosis and treatment of subacromial pain syndrome. Acta Orthop [Internet]. 2014;85(3):314-22.

22. Physiotherapy evidence database (PEDro) website (Jan 2016) http://www.pedro.org.au/english/downloads/pedro-scale/

23. Maher CG, Sherrington C, Herbert RD, Moseley AM, Elkins M. Reliability of the PEDro scale for rating quality of randomized controlled trials. Phys Ther. 2003;83(8):713-21.

24. Sherrington C, Herbert RD, Maher CG, Moseley a M. PEDro. A database of randomized trials and systematic reviews in physiotherapy. Man Ther. 2000;5(4):2236.

25. de Morton NA. The PEDro scale is a valid measure of the methodological quality of clinical trials: a demographic study. Aust J Physiother [Internet]. Elsevier; 2009;55(2):129-33.

26. Moseley AM, Herbert RD, Sherrington C, Maher CG. Evidence for physiotherapy practice: A survey of the Physiotherapy Evidence Database (PEDro). Aust J Physiother [Internet]. Australian Physiotherapy Association; 2002;48(1):43-9.

27. Shah M, Sutaria J, Khant A. Effectiveness of scapular stability exercises in the patient with the shoulder impingement syndrome. Indian Journal of Physical Therapy 2(1):79-84.

28. Wallace BC, Dahabreh IJ, Trikalinos T a, Lau J, Trow P, Schmid CH. Closing the Gap between Methodologists and End-Users: $R$ as a Computational Back-End. Wiley Interdiscip Rev Comput. 2012;49(5):1-15.

29. DerSimonian R, Laird N. Meta-analysis in clinical trials. Control Clin Trials. 1986;7(3):177-88. 
30. Higgins JPT, Thompson SG, Deeks JJ, Altman DG. Measuring inconsistency in metaanalyses. BMJ Br Med J. 2003;327(7414):557-60.

31. Smith BE, Littlewood C, May S. An update of stabilisation exercises for low back pain: a systematic review with meta-analysis. BMC Musculoskelet Disord [Internet]. 2014;15:416.

32. Baskurt Z, Baskurt F, Gelecek N, Ozkan MH. The effectiveness of scapular stabilisation exercises in the patients with subacromial impingement syndrome. Journal of Back and Musculoskeletal Rehabilitation. 2011;24:173-9.

33. Deeks, J. J., Higgins, J. P. and Altman, D. G. (2008) Analysing Data and Undertaking Meta-Analyses, in Cochrane Handbook for Systematic Reviews of Interventions: Cochrane Book Series (eds J. P. Higgins and S. Green), John Wiley \& Sons, Ltd, Chichester, UK. doi: 10.1002/9780470712184.ch9

34. Van Tulder MW, Assendelft WJ, Koes BW, Bouter LM. Method guidelines for systematic reviews in the Cochrane Collaboration Back Review Group for Spinal Disorders. Spine (Phila Pa 1976). 1997;22(20):2323-30.

35. Struyf F, Nijs J, Mollekens S, Jeurissen I, Truijen S, Mottram S, et al. Scapularfocused treatment in patients with shoulder impingement syndrome: A randomized clinical trial. Clin Rheumatol. 2013;32(1):73-85.

36. Moezy A, Sepehrifar S, Solaymani Dodaran M. The effects of scapular stabilization based exercise therapy on pain, posture, flexibility and shoulder mobility in patients with shoulder impingement syndrome: a controlled randomized clinical trial. Med $\mathrm{J}$ Islam Repub Iran [Internet]. 2014;28(2):87.

37. Littlewood C. Scapular-focused treatment in patients with shoulder impingement syndrome: A randomised clinical trial. Clin Rheumatol. 2013;32(3):417.

38. Littlewood C, Malliaras P, Mawson S, May S, Walters S. Patients with rotator cuff tendinopathy can successfully self-manage, but with certain caveats: A qualitative study. Physiother; 2014;100(1):80-5.

39. Littlewood C, Mawson S, May S, Walters S. Understanding the barriers and enablers to implementation of a self-managed exercise intervention: A qualitative study. Physiother; 2015;101(3):279-85.

40. Mulligan EP, Huang M, Dickson T, Khazzam M. the Effect of Axioscapular and Rotator Cuff Exercise Training Sequence in Patients With Subacromial Impingement Syndrome: a Randomized Crossover Trial. Int J Sports Phys Ther [Internet]. 2016;11(1):94-107.

41. Ellenbecker TS, Kibler W Ben, Bailie DS, Caplinger R, Davies GJ, Riemann BL. Reliability of scapular classification in examination of professional baseball players. Clin Orthop Relat Res. 2012;470(6):1540-4.

42. Ratcliffe E, Pickering S, McLean S, Lewis J. Is there a relationship between subacromial impingement syndrome and scapular orientation? A systematic review. Br J Sports Med [Internet]. 2013;1251-6.

43. Camargo PR, Alburquerque-Sendín F, Avila M a, Haik MN, Vieira A, Salvini TF. Effects of Stretching and Strengthening Exercises With and Without Manual Therapy on Scapular Kinematics, Function, and Pain in Individuals With Shoulder Impingement: A Randomized Controlled Trial. J Orthop Sports Phys Ther [Internet]. 
2015;58(12):1-34.

44. Lefebvre, C., Manheimer, E. and Glanville, J. (2008) Searching for Studies, in Cochrane Handbook for Systematic Reviews of Interventions: Cochrane Book Series (eds J. P. Higgins and S. Green), John Wiley \& Sons, Ltd, Chichester, UK. doi: 10.1002/9780470712184.ch6

45. Van Driel ML, De Sutter A, De Maeseneer J, Christiaens T. Searching for unpublished trials in Cochrane reviews may not be worth the effort. J Clin Epidemiol [Internet]. Elsevier Inc; 2009;62(8):838-44.e3. 